\title{
Asymptotic Stability of Neutral Set-Valued Functional Differential Equation by Fixed Point Method
}

\author{
Junyan Bao $i$ and Peiguang Wang $(\mathbb{D}$ \\ College of Mathematics and Information Science, Hebei University, Baoding 071002, China \\ Correspondence should be addressed to Peiguang Wang; pgwang@hbu.edu.cn
}

Received 28 February 2020; Accepted 4 April 2020; Published 27 April 2020

Guest Editor: Abdul Qadeer Khan

Copyright (c) 2020 Junyan Bao and Peiguang Wang. This is an open access article distributed under the Creative Commons Attribution License, which permits unrestricted use, distribution, and reproduction in any medium, provided the original work is properly cited.

This paper studies a class of nonlinear neutral set-valued functional differential equations. The globally asymptotic stability theorem with necessary and sufficient conditions is obtained via the fixed point method. Meanwhile, we give an example to illustrate the obtained result.

\section{Introduction}

It is well known that Lyapunov's direct method is an important technique to consider the stability of various differential equations. However, this method is not always valid for stability analysis in the functional differential equation when the delay is unbounded or when the equation has unbounded terms [1-3]. Burton and many researchers found a way to these difficulties by using various fixed point theorems; we can refer to the literature studies [4-14].

Recently, the study of qualitative analysis of the setvalued differential equation has attracted much attention. The stability results of various set-valued differential equations were obtained by applying Lyapunov's direct method. The results can be found in the monograph [15], the papers for the set-valued differential equation [16-24], setvalued functional differential equation [25-29], and other equations [6, 9, 30-32]. However, according to what we know so far, there are few stability results for the set-valued differential equation via the fixed point method. Inspired by the application of the fixed point method mentioned above, in this paper, we study a class of nonlinear neutral set-valued functional differential equations:

$$
\left\{\begin{array}{l}
D_{H} X(t)=-a(t) X(t)+b(t) D_{H} X(t-\tau) \\
\quad+F(t, X(t), X(t-\tau)), \quad t \in I, \\
X(t)=\Phi(t), \quad t \in I_{0},
\end{array}\right.
$$

where $X \in C^{1}\left(I, K_{c}(\mathbb{E})\right) \cup C\left(I_{0}, K_{c}(\mathbb{E})\right), F \in C\left(I \times K_{c}(\mathbb{E}) \times\right.$ $\left.K_{c}(\mathbb{E}), K_{c}(\mathbb{E})\right), \quad$ and $\quad F(t, \theta, \theta)=\theta ; \quad \Phi \in C\left(I_{0}, K_{c}(\mathbb{E})\right)$, $a, b \in C(I, \mathbb{R}), \quad I=\left[t_{0},+\infty\right)$, and $I_{0}=\left[t_{0}-\tau, t_{0}\right] . K_{c}(\mathbb{E})$ denote the collection of all nonempty, compact convex subsets of Banach space $\mathbb{E}$; $\theta$ denotes the null set-valued function $\theta: I \longrightarrow K_{c}(\mathbb{E})$, and $\theta(t)=\{0\}$ for $t \in I$, $\tau>0$ is a constant.

The aim of this paper is to obtain an asymptotic stability theorem with a necessary and sufficient condition via the fixed point method. In addition, an application of the main result is presented.

\section{Preliminaries}

To get the desired result, we first give some notations, definitions, and propositions briefly; for the details, see the literature [15].

Let $K_{c}(\mathbb{E})$ denote the collection of all nonempty, compact convex subsets of Banach space $\mathbb{E}$, given $A, B \in K_{c}(\mathbb{E})$, defining the Hausdorff metric between $A$ and $B$ as follows:

$$
D[A, B]=\max \left\{\sup _{b \in B} d(b, A), \sup _{a \in A} d(a, B)\right\},
$$

where $\quad d(b, A)=\inf \{d(b, a): a \in A\} \quad$ and $\quad d(a, B)=$ $\inf \{d(a, b): b \in B\}$. The Hausdorff metric satisfies the properties as follows: 


$$
\begin{aligned}
D[A, B] & \geq 0 \text { with } D[A, B]=0 \text { if and only if } A=B, \\
D[A, B] & =D[B, A], \\
D[\lambda A, \lambda B] & =|\lambda| D[A, B], \\
D[A, B] & \leq D[A, C]+D[C, B],
\end{aligned}
$$

for all $A, B, C \in K_{c}(\mathbb{E})$ and $\lambda \in \mathbb{R}$.

In the sense of the above metric $D$, the set $K_{c}(\mathbb{E})$ is a complete metric space.

Definition 1 (see [15]). The set-valued function $F:[a, b]$ $\longrightarrow K_{c}(\mathbb{E})$ is Hukuhara differentiable at $t_{0} \in[a, b]$ if the limits

$$
\begin{aligned}
& \lim _{h \longrightarrow 0^{+}} \frac{F\left(t_{0}+h\right)-F\left(t_{0}\right)}{h}, \\
& \lim _{h \longrightarrow 0^{+}} \frac{F\left(t_{0}\right)-F\left(t_{0}-h\right)}{h},
\end{aligned}
$$

exist in $K_{c}(\mathbb{E})$ and equal to $D_{H} F\left(t_{0}\right) . D_{H} F\left(t_{0}\right) \in K_{c}(\mathbb{E})$ is called the Hukuhara derivative of $F(t)$ at $t_{0} \in[a, b]$.

By embedding $K_{c}(\mathbb{E})$ as a complete cone in a corresponding Banach space and taking into account the differentiation of the Bochner integral, we can find that if

$$
F(t)=X_{0}+\int_{a}^{t} \Psi(s) \mathrm{d} s, \quad X_{0} \in K_{c}(\mathbb{E}),
$$

then $D_{H} F(t)$ exists and $D_{H} F(t)=\Psi(t)$ a.e. on $[a, b]$ holds, where $\Psi:[a, b] \longrightarrow K_{c}(\mathbb{E})$ is integrable in the sense of Bochner.

Proposition 1 (see [15]). Let $X:[a, b] \longrightarrow K_{c}(\mathbb{E})$ be Hukuhara differentiable and have continuous Hukuhara derivative $D_{H} X$ on $[a, b]$; then, we have

$$
X(t)=X\left(t_{0}\right)+\int_{t_{0}}^{t} D_{H} X(t) \mathrm{d} t, \quad a \leq t_{0} \leq t \leq b .
$$

Proposition 2 (see [15]). If $X, Y:[a, b] \longrightarrow K_{c}(\mathbb{E})$ is integrable, then

$$
D\left[\int_{t_{0}}^{t} X(s) \mathrm{d} s, \int_{t_{0}}^{t} Y(s) \mathrm{d} s\right] \leq \int_{t_{0}}^{t} D[X(s), Y(s)] \mathrm{d} s .
$$

For each $\left(t_{0}, \Phi\right) \in I \times C\left(I_{0}, K_{c}(\mathbb{E})\right), X(t)$ is said to be a solution of $(1)$ through $\left(t_{0}, \Phi\right)$ if $X \in C^{1}\left(I, K_{c}(\mathbb{E})\right) \cup$ $C\left(I_{0}, K_{c}(\mathbb{E})\right)$ satisfies $(1)$ on $I$ and $X(t)=\Phi(t)$ on $I_{0}$, and we denote the solution $X(t)$ by $X\left(t, t_{0}, \Phi\right)$.

Let $\mathscr{X}=C^{1}\left(I_{0} \cup I, K_{c}(\mathbb{E})\right)$ and $\mathscr{I}=C^{1}\left(I_{0}, K_{c}(\mathbb{E})\right)$ with norm

$$
\begin{aligned}
\|X\| & =\sup _{t \in I} D[X(t), \theta], \\
\|\Phi\| & =\max _{t \in I_{0}} D[\Phi(t), \theta],
\end{aligned}
$$

respectively, and $\|X\|_{0}=\max \left\{\|X\|,\left\|D_{H} X\right\|\right\}$. By the properties of Hausdorff metric $D$ and the definition of $\|\cdot\|_{0}$, we can get the following: (i) $\|X(t)\|_{0} \geq 0$, and $\|X(t)\|_{0}=0$ if and only if $X(t)=\theta$,

(ii) $\|X(t)+Y(t)\|_{0} \leq\|X(t)\|_{0}+\|Y(t)\|_{0}$,

(iii) $|\lambda X(t)|=|\lambda X(t)|$

where $X(t), Y(t) \in \mathscr{X}$ and $\lambda \in \mathbb{R}$.

Definition 2. The trivial solution of (1) is said to be

(i) Stable in $C^{1}$ if for any $t_{0} \in \mathbb{R}^{+}$and $\varepsilon>0$, there exists $\delta=\delta\left(\varepsilon, t_{0}\right)$ such that $\Phi \in \mathscr{I}$, and $\|\Phi\|_{0}<\delta$ implies that $\|X\|_{0}<\varepsilon$ and $t \geq t_{0}$.

(ii) Globally asymptotically stable in $C^{1}$ if it is stable, and for any $t_{0} \in I, \Phi \in \mathscr{I}$ implies that

$\lim _{t \longrightarrow+\infty} X\left(t, t_{0}, \Phi\right)=\lim _{t \longrightarrow+\infty} D_{H} X\left(t, t_{0}, \Phi\right)=\{0\}$.

For $A, B \in K_{c}(\mathbb{E})$ and $\lambda \in \mathbb{R}$, we can define the following addition and scalar multiplication as follows: $A+B=\{a+b: a \in A, b \in B\}, \lambda A=\{\lambda a: a \in A\}$. Then, with the algebraic operations of addition and nonnegative scalar multiplication, $\left(K_{c}(\mathbb{E}), D\right)$ becomes a semilinear metric space.

In order to apply the fixed point method, we first need to prove the following lemma.

Lemma 1. Let $\mathscr{X}_{0}=\left\{X(t) \in \mathscr{X}: \lim _{t \rightarrow+\infty} X(t)=\right.$ $\left.\lim _{t \longrightarrow+\infty} D_{H} X(t)=\{0\}\right\}$. Then, $\left(\mathscr{X}_{0},\|\cdot\|_{0}\right)$ is a semilinear complete metric space.

Proof. Firstly, from the previous discussion, we know that $\left(\mathscr{X}_{0},\|\cdot\|_{0}\right)$ is a semilinear metric space. Next, we prove the space is complete. Assume that $\left\{X_{m}(t)\right\}$ is a Cauchy sequence; then, for any $\varepsilon>0$, there exists positive integer $N$ such that, for all $m, n>N$, we have $\left\|X_{n}(t)-X_{m}(t)\right\|_{0}<\varepsilon$, $t \geq t_{0}-\tau$. Hence,

$$
\begin{aligned}
& D\left[X_{m}(t), X_{n}(t)\right] \leq\left\|X_{m}(t)-X_{n}(t)\right\|_{0}<\varepsilon, \quad t \geq t_{0}-\tau, \\
& D\left[D_{H} X_{m}(t), D_{H} X_{n}(t)\right] \leq\left\|X_{m}(t)-X_{n}(t)\right\|_{0}<\varepsilon, \quad t \geq t_{0}-\tau .
\end{aligned}
$$

Then, $\left\{X_{n}(t)\right\}$ and $\left\{D_{H} X_{n}(t)\right\}$ are Cauchy sequences in $K_{c}(\mathbb{E})$, respectively. Since $\left(K_{c}(\mathbb{E}), D\right)$ is a complete metric space, there exist set-valued functions $X(t)$ and $Z(t)$ such that

$$
\lim _{m \longrightarrow \infty} X_{m}(t)=X(t), \quad \lim _{m \longrightarrow \infty} D_{H} X_{m}(t)=Z(t) .
$$

First, we prove that $X(t)$ is a continuous function on $I_{0} \cup I$. From (11), there exists a positive integer $M$ such that $D\left[X_{m}(t), X(t)\right]<(\varepsilon / 3)$ for all $m>M$ and $t \geq t_{0}-\tau$. In addition, since $X_{m}(t)$ is a continuous set-valued function, there exists $\delta>0$ such that $|t-s|<\delta$ implies that $D\left[X_{m}(t), X_{m}(s)\right]<(\varepsilon / 3)$ for $s, t \geq t_{0}-\tau$. Then, for any $t \geq t_{0}-\tau$ with $|t-s|<\delta$ and $m>M$, we have

$$
\begin{aligned}
D[X(t), X(s)] \leq & D\left[X(t), X_{m}(t)\right]+D\left[X_{m}(t), X_{m}(s)\right] \\
& +D\left[X_{m}(s), X(s)\right]<\varepsilon .
\end{aligned}
$$


Using the same way, we can also prove that $Z(t)$ is a continuous function.

Next, we prove that $D_{H} X(t)=Z(t)$. In fact, for any $t_{0}, t \in I$ and $t>t_{0}$, we have

$$
X_{m}(t)=X_{m}\left(t_{0}\right)+\int_{t_{0}}^{t} D_{H} X_{m}(s) \mathrm{d} s .
$$

By (11) and (13), $X(t)=X\left(t_{0}\right)+\int_{t_{0}}^{t} Z(s) \mathrm{d} s$ holds when $m \longrightarrow \infty$. Using Proposition 1, we get $D_{H} X(t)=Z(t)$. Meanwhile, we can prove that $\lim _{t \rightarrow+\infty} X(t)=$ $\lim _{t \longrightarrow+\infty} Z(t)=\{0\}$. Therefore, $X(t) \in \mathscr{X}_{0}$. This completes the proof of Lemma 1.

\section{Main Result}

In this section, we establish the necessary and sufficient conditions for the global asymptotic stability of trivial solution of equation (1) by using the fixed point method.

Theorem 1. Assume that the following conditions hold:

$\left(A_{1}\right)$ : the function $a(t)$ is bounded and $\lim _{t \longrightarrow+\infty} \inf \int_{t_{0}}^{t} a(s) d s>-\infty$.

$\left(A_{2}\right)$ : there exist functions $\lambda_{1}, \lambda_{2} \in C\left(I, \mathbb{R}^{+}\right)$such that

$$
\begin{aligned}
& \left\|F\left(t, X_{1}, Y_{1}\right)-F\left(t, X_{2}, Y_{2}\right)\right\| \\
& \quad \leq \lambda_{1}(t)\left\|X_{1}-X_{2}\right\|+\lambda_{2}(t)\left\|Y_{1}-Y_{2}\right\| .
\end{aligned}
$$

$\left(A_{3}\right)$ : there exists a constant $\alpha \in(0,1)$ such that

$$
\begin{aligned}
& \int_{t_{0}}^{t} e^{-\int_{s}^{t} a(v) \mathrm{d} v}\left[|b(s)|+\lambda_{1}(s)+\lambda_{2}(s)\right] \mathrm{d} s \leq \alpha, \\
& |a(t)| \int_{t_{0}}^{t} e^{-\int_{s}^{t} a(v) d v}\left[|b(s)|+\lambda_{1}(s)+\lambda_{2}(s)\right] \mathrm{d} s \\
& \quad+|b(t)|+\lambda_{1}(t)+\lambda_{2}(t) \leq \alpha .
\end{aligned}
$$

Then, the trivial solution of equation (1) is globally asymptotically stable in $C^{1}$ if and only if

$$
\int_{t_{0}}^{+\infty} a(s) \mathrm{d} s=+\infty
$$

Proof. We will prove this conclusion in two steps.

3.1. Proof of Sufficiency. Let $\mathscr{D}=\left\{X \in \mathscr{X}_{0}: X(t)=\right.$ $\left.\Phi(t), t \in I_{0}\right\}$. Then, $\mathscr{D}$ is a nonempty, closed subset of $\mathscr{X}_{0}$. At the same time, we can get equation (1) with initial condition $X\left(t_{0}\right)=\Phi(t)$ which is equivalent to the following integral equation:

$$
\left\{\begin{array}{l}
X(t)=\Phi\left(t_{0}\right) e^{-\int_{t_{0}}^{t} a(v) \mathrm{d} v}+\int_{t_{0}}^{t} e^{-\int_{s}^{t} a(v) \mathrm{d} v}\left[b(s) D_{H} X(s-\tau)\right. \\
\quad+F(s, X(s), X(s-\tau))] \mathrm{d} s, t \in I, \\
X(t)=\Phi(t), t \in I_{0} .
\end{array}\right.
$$

We define the mapping Q: $\mathscr{D} \longrightarrow C^{1}\left(I_{0} \cup I, K_{c}(\mathbb{E})\right)$ as follows:

$$
(Q X)(t)=\left\{\begin{array}{l}
\Phi\left(t_{0}\right) e^{-\int_{t_{0}}^{t} a(v) \mathrm{d} v}+\int_{t_{0}}^{t} e^{-\int_{s}^{t} a(v) \mathrm{d} v}\left[b(s) D_{H} X(s-\tau)\right. \\
\quad+F(s, X(s), X(s-\tau))] \mathrm{d} s, t \in I, \\
\Phi(t), t \in I_{0} .
\end{array}\right.
$$

Firstly, we prove that $Q$ is a mapping of $\mathscr{D} \longrightarrow \mathscr{D}$. In fact, we just need to prove that $(Q X)(t) \longrightarrow\{0\}$ and $D_{H}(Q X)(t) \longrightarrow\{0\}$ as $t \longrightarrow+\infty$. Since $\lim _{t \longrightarrow+\infty} X(t)=$ $\lim _{t \longrightarrow+\infty} D_{H} X(t)=\{0\}$, for any $\varepsilon>0$, there exists $T>0$ such that $t>T$ implies

$$
\max \left\{\|X(t)\|,\|X(t-\tau)\|,\left\|D_{H} X(t-\tau)\right\|\right\}<\varepsilon .
$$

Thus, from (18), (19), $\left(A_{2}\right)$, and $\left(A_{3}\right)$, we have

$$
\begin{aligned}
\|(Q X)(t)\| \leq & \left\|\Phi\left(t_{0}\right)\right\| e^{-\int_{t_{0}}^{t} a(v) \mathrm{d} v} \\
& +\int_{t_{0}}^{T} e^{-\int_{s}^{t} a(v) \mathrm{d} v}\left\|b(s) D_{H} X(s-\tau)+F(s, X(s), X(s-\tau))\right\| \mathrm{d} s \\
& +\int_{T}^{t} e^{-\int_{s}^{t} a(v) \mathrm{d} v}\left\|b(s) D_{H} X(s-\tau)+F(s, X(s), X(s-\tau))-F(s, \theta, \theta)\right\| \mathrm{d} s \leq e^{-\int_{t_{0}}^{t} a(v) d v}\left[\left\|\Phi\left(t_{0}\right)\right\|\right. \\
& \left.+\int_{t_{0}}^{T} e^{\int_{t_{0}}^{s} a(v) d v}\left\|b(s) D_{H} X(s-\tau)+F(s, X(s), X(s-\tau))\right\| \mathrm{d} s\right] \\
& +\int_{T}^{t} e^{-\int_{s}^{t} a(v) \mathrm{d} v}\left[\mid b(s)\left\|D_{H} X(s-\tau)\right\|+\lambda_{1}(s)\|X(s)\|+\lambda_{2}(s)\|X(s-\tau)\|\right] \mathrm{d} s
\end{aligned}
$$




$$
\begin{aligned}
& \leq e^{-\int_{t_{0}}^{t} a(v) \mathrm{d} v}\left[\left\|\Phi\left(t_{0}\right)\right\|+\int_{t_{0}}^{T} e^{\int_{t_{0}}^{s} a(v) \mathrm{d} v}\left\|b(s) D_{H} X(s-\tau)+F(s, X(s), X(s-\tau))\right\| \mathrm{d} s\right] \\
& \quad+\varepsilon \int_{T}^{t} e^{-\int_{s}^{t} a(v) \mathrm{d} v}\left[|b(s)|+\lambda_{1}(s)+\lambda_{2}(s)\right] \mathrm{d} s \\
& \leq e^{-\int_{t_{0}}^{t} a(v) \mathrm{d} v}\left[\left\|\Phi\left(t_{0}\right)\right\|+\int_{t_{0}}^{T} e^{\int_{t_{0}}^{s} a(v) \mathrm{d} v}\left\|b(s) D_{H} X(s-\tau)+F(s, X(s), X(s-\tau))\right\| \mathrm{d} s\right]+\alpha \varepsilon, t>T .
\end{aligned}
$$

Then, by condition (16), there exists $T_{1}>\mathrm{T}$ such that, for $t>T_{1}$ implies

$$
\begin{aligned}
& e^{-\int_{t_{0}}^{t} a(v) \mathrm{d} v}\left[\left\|\Phi\left(t_{0}\right)\right\|+\int_{t_{0}}^{T} e^{\int_{t_{0}}^{s} a(v) \mathrm{d} v} \| b(s) D_{H} X(s-\tau)\right. \\
& +F(s, X(s), X(s-\tau)) \| \mathrm{d} s]<\varepsilon .
\end{aligned}
$$

Thus, we have $\|(Q X)(t)\|<(1+\alpha) \varepsilon$ for $t>T_{1}$, and we can obtain $(Q X)(t) \longrightarrow\{0\}$ as $t \longrightarrow+\infty$. In addition, we can also get

$$
\begin{aligned}
D_{H}(Q X)(t)= & -a(t) \Phi\left(t_{0}\right) e^{-\int_{t_{0}}^{t} a(v) \mathrm{d} v} \\
& -a(t) \int_{t_{0}}^{t} e^{-\int_{s}^{t} a(v) \mathrm{d} v}\left[b(s) D_{H} X(s-\tau)\right. \\
& +F(s, X(s), X(s-\tau))] \mathrm{d} s \\
& +\left[b(t) D_{H} X(t-\tau)+F(t, X(t), X(t-\tau))\right] \\
= & -a(t)(Q X)(t)+b(t) D_{H} X(t-\tau) \\
& +F(t, X(t), X(t-\tau)) \\
= & -a(t)(Q X)(t)+D_{H} X(t)+a(t) X(t) .
\end{aligned}
$$

Furthermore, from (22), we can get

$\left\|D_{H}(Q X)(t)\right\| \leq|a(t)|\|(Q X)(t)\|+\left\|D_{H} X(t)\right\|+|a(t)|\|X(t)\|$.

From $\left(\mathrm{A}_{1}\right)$ and $\lim (Q X)(t)=\lim X(t)=\{0\}$, we have $\lim _{t \longrightarrow+\infty} D_{H}\left(\vec{Q}^{t} \overrightarrow{Q X}^{\dagger}\right)(t)=\{0\}$ for $\vec{X}^{t} \in \mathscr{D}$. Therefore, $(Q X)(t) \in \mathscr{D}$ for $X \in \mathscr{D}$, that is, $Q: \mathscr{D} \longrightarrow \mathscr{D}$.

Secondly, we prove that the mapping $Q$ has a unique fixed point.

Let $X, Y \in \mathscr{D}$, and by (18), $\left(\mathrm{A}_{2}\right)$, and $\left(\mathrm{A}_{3}\right)$, we have

$$
\begin{aligned}
& \|(Q X)(t)-(Q Y)(t)\| \\
& \leq \int_{t_{0}}^{t} e^{-\int_{s}^{t} a(v) \mathrm{d} v}\left[|b(s)|\left\|D_{H} X(s)-D_{H} Y(s)\right\|\right. \\
& \quad+\|F(s, X(s), X(s-\tau))-F(s, Y(s), Y(s-\tau))\|] \mathrm{d} s \\
& \leq\|X(t)-Y(t)\|_{0} \int_{t_{0}}^{t} e^{-\int_{s}^{t} a(v) \mathrm{d} v}\left[|b(s)|+\lambda_{1}(s)+\lambda_{2}(s)\right] \mathrm{d} s \\
& \leq \alpha\|X(t)-Y(t)\|_{0}, \quad \alpha<1, t \geq t_{0} .
\end{aligned}
$$

Similarly, from (18), (24), $\left(\mathrm{A}_{2}\right)$, and $\left(\mathrm{A}_{3}\right)$, we can also get

$$
\begin{aligned}
& \left\|D_{H}(Q X)(t)-D_{H}(Q Y)(t)\right\| \\
& \leq|a(t)|\|(Q X)(t)-(Q Y)(t)\| \\
& \quad+|b(t)|\left\|D_{H} X(t)-D_{H} Y(t)\right\| \\
& \quad+\|F(t, X(t), X(t-\tau))-F(t, Y(t), Y(t-\tau))\| \\
& \leq\|X(t)-Y(t)\|_{0}\left\{| a ( t ) | \int _ { t _ { 0 } } ^ { t } e ^ { - \int _ { s } ^ { t } a ( v ) \mathrm { d } v } \left[|b(s)|+\lambda_{1}(s)\right.\right. \\
& \left.\left.\quad+\lambda_{2}(s)\right] \mathrm{d} s+|b(t)|+\lambda_{1}(t)+\lambda_{2}(t)\right\} \\
& \leq \alpha\|X(t)-Y(t)\|_{0}, \quad t \geq t_{0} .
\end{aligned}
$$

By (24) and (25), we know that $Q$ is a contraction mapping. Therefore, using the principle of contraction mapping, $Q$ has a unique fixed point $X \in \mathscr{D}$ which is a unique solution of equation (1) and satisfies

$$
\lim _{t \longrightarrow+\infty} X(t)=\lim _{t \longrightarrow+\infty} D_{H} X(t)=\{0\} .
$$

Finally, we prove the global asymptotic stability of equation (1). To do this, we first prove that the trivial solution of (1) is stable. Let

$$
\begin{aligned}
& M_{1}=\sup _{t \in I}\left\{e^{-\int_{t_{0}}^{t} a(v) \mathrm{d} v}\right\}, \\
& M_{2}=\sup _{t \in I}\{|a(t)|\} .
\end{aligned}
$$

For any $\varepsilon>0$, we choose $\delta>0$ satisfying $\max \left\{\delta, M_{1} \delta+\alpha \varepsilon, M_{1} M_{2} \delta+\alpha \varepsilon\right\}<\varepsilon$. If $X(t)=X\left(t, t_{0}, \Phi\right)$ is a solution of equation (1) with $\|\Phi(t)\|_{0}<\delta$, we can claim that $\|X(t)\|_{0}<\varepsilon$ on $t \in I$. In fact, if this is not true and we notice that $\|X(t)\|_{0}<\varepsilon$ on $I_{0}$, then there exists $\bar{t}>t_{0}$ such that

$$
\begin{aligned}
& \max \left\{\|X(\bar{t})\|,\left\|D_{H} X(\bar{t})\right\|\right\}=\varepsilon, \\
& \max \left\{\|X(t)\|,\left\|D_{H} X(t)\right\|\right\}<\varepsilon, \quad \text { for } t \in\left[t_{0}-\tau, \bar{t}\right) .
\end{aligned}
$$


If $\|X(\bar{t})\|=\varepsilon$, then it follows from $\left(\mathrm{A}_{2}\right)$ and $\left(\mathrm{A}_{3}\right)$ that

$$
\begin{aligned}
\|X(\bar{t})\| \leq & \left\|\Phi\left(t_{0}\right)\right\| e^{-\int_{t_{0}}^{\bar{t}} a(v) \mathrm{d} v} \\
& +\int_{t_{0}}^{\bar{t}} e^{-\int_{s}^{\bar{t}} a(v) \mathrm{d} v} \| b(s) D_{H} X(s-\tau) \\
& +F(s, X(s), X(s-\tau)) \| \mathrm{d} s \\
\leq & M_{1} \delta+\varepsilon \int_{t_{0}}^{\bar{t}} e^{-\int_{s}^{\bar{t}} a(v) \mathrm{d} v}\left[|b(s)|+\lambda_{1}(s)+\lambda_{2}(s)\right] \mathrm{d} s \\
\leq & M_{1} \delta+\alpha \varepsilon<\varepsilon,
\end{aligned}
$$

which contradicts the definition of $\bar{t}$.

If $\left\|D_{H} X(\bar{t})\right\|=\varepsilon$, then it follows from $\left(\mathrm{A}_{2}\right)$ and $\left(\mathrm{A}_{3}\right)$ that

$$
\begin{aligned}
\left\|D_{H} X(\bar{t})\right\| \\
\leq|a(\bar{t})|\left\|\Phi\left(t_{0}\right)\right\| e^{-\int_{t_{0}}^{\bar{t}} a(v) \mathrm{d} v}+|b(\bar{t})|\left\|D_{H} X(\bar{t}-\tau)\right\| \\
\quad+\|F(\bar{t}, X(\bar{t}), X(\bar{t}-\tau))\| \\
\quad+|a(\bar{t})| \int_{t_{0}}^{\bar{t}} e^{-\int_{s}^{\bar{t}} a(v) d v} \| b(s) D_{H} X(s-\tau) \\
\quad+F(s, X(s), X(s-\tau)) \| \mathrm{d} s \\
\leq M_{1} M_{2} \delta+\varepsilon\left\{|a(t)| \int_{t_{0}}^{t} e^{-\int_{s}^{t} a(v) \mathrm{d} v}\left[|b(s)|+\lambda_{1}(s)+\lambda_{2}(s)\right] \mathrm{d} s\right. \\
\leq M_{1} M_{2} \delta+\alpha \varepsilon<\varepsilon,
\end{aligned}
$$

which also contradicts the definition $\bar{t}$. Hence, $\|X(t)\|_{0}<\varepsilon$ for all $t \geq t_{0}$, and the trivial solution of (1) is stable. This, together with (26), claims that the trivial solution of (1) is globally asymptotically stable.
3.2. Proof of Necessity. Assume that (16) is not valid. Then, by condition $\left(A_{1}\right)$, there exists a sequence $\left\{t_{n}\right\}$ such that $\lim _{n \longrightarrow+\infty} \int_{t_{0}}^{t_{n}} a(v) \mathrm{d} v=l$ for some $l \in R$, where $t_{n} \longrightarrow+\infty$ as $n \longrightarrow \infty$. Furthermore, we also choose a positive constant $p$ satisfying

$$
-p \leq \int_{t_{0}}^{t_{n}} a(v) \mathrm{d} v \leq p, \quad \text { for } \quad n \geq 1 .
$$

By $\left(\mathrm{A}_{3}\right)$, we have

$$
I_{n}=\int_{t_{0}}^{t_{n}} e^{\int_{t_{0}}^{s} a(v) \mathrm{d} v}\left[|b(s)|+\lambda_{1}(s)+\lambda_{2}(s)\right] \mathrm{d} s \leq \alpha e^{\int_{t_{0}}^{t_{n}} a(v) \mathrm{d} v},
$$

that is, the sequence of $\left\{I_{n}\right\}$ is bounded. Thus, there exists a convergent subsequence. For convenience, we may assume that $\lim _{n \longrightarrow+\infty} I_{n}=\lambda$. Let

$$
\begin{aligned}
& K=\sup _{t \in I}\left\{e^{-\int_{t_{0}}^{t} a(v) \mathrm{d} v}\right\}, \\
& A=\sup _{t \in I}\{|a(t)|\} .
\end{aligned}
$$

Then, there exists a sufficient large positive integer $k$ such that, for all $n>k$,

$$
\left|I_{n}-I_{k}\right|=\int_{t_{k}}^{t_{n}} e^{\int_{t_{0}}^{s} a(v) \mathrm{d} v}\left[|b(s)|+\lambda_{1}(s)+\lambda_{2}(s)\right] \mathrm{d} s<\frac{1-\alpha}{4 M e^{2 p}},
$$

where $M=\max \left\{K e^{p}, K A e^{p}\right\}$.

For any $\delta>0$, we consider the solution $X\left(t, t_{k}, \Phi\right)$ of (1) with $\left\|\Phi\left(t_{k}\right)\right\|>(1 / 2) \delta$ and $\|\Phi(t)\|<\delta, \quad t \in\left[t_{k}-\tau, t_{k}\right]$. It follows from (17), (22), (31), $\left(\mathrm{A}_{2}\right)$, and $\left(\mathrm{A}_{3}\right)$ that

$$
\begin{aligned}
\|X(t)\| \leq & \left\|\Phi\left(t_{k}\right)\right\| e^{-\int_{t_{k}}^{t} a(v) \mathrm{d} v} \\
& +\int_{t_{k}}^{t} e^{-\int_{s}^{t} a(v) \mathrm{d} v}\left\|b(s) D_{H} X(s-\tau)+F(s, X(s), X(s-\tau))\right\| \mathrm{d} s \\
\leq & \left\|\Phi\left(t_{k}\right)\right\| e^{-\int_{t_{0}}^{t} a(v) \mathrm{d} v} e^{\int_{t_{0}}^{t_{k}} a(v) \mathrm{d} v} \\
& +\|X(t)\|_{0 k} \int_{t_{k}}^{t} e^{-\int_{s}^{t} a(v) d v}\left[|b(s)|+\lambda_{1}(s)+\lambda_{2}(s)\right] \mathrm{d} s \\
\leq & K e^{p} \delta+\alpha\|X(t)\|_{0 k}, \quad t \in\left[t_{k},+\infty\right),
\end{aligned}
$$




$$
\begin{aligned}
\left\|D_{H} X(t)\right\| \leq & |a(t)|\left\|\Phi\left(t_{k}\right)\right\| e^{-\int_{t_{k}}^{t} a(v) \mathrm{d} v}+|b(t)|\left\|D_{H} X(t-\tau)\right\|+\|F(t, X(t), X(t-\tau))\| \\
& +|a(t)| \int_{t_{k}}^{t} e^{-\int_{s}^{t} a(v) \mathrm{d} v}\left\|b(s) D_{H} X(s-\tau)+F(s, X(s), X(s-\tau))\right\| \mathrm{d} s \\
\leq & |a(t)|\left\|\Phi\left(t_{k}\right)\right\| e^{-\int_{t_{0}}^{t} a(v) \mathrm{d} v} e^{\int_{t_{0}}^{t_{k}} a(v) \mathrm{d} v}+\|X(t)\|_{0 k}\left[|b(t)|+\lambda_{1}(t)+\lambda_{2}(t)\right] \\
& +\|X(t)\|_{0 k} \int_{t_{k}}^{t} e^{-\int_{s}^{t} a(v) \mathrm{d} v}\left[|b(s)|+\lambda_{1}(s)+\lambda_{2}(s)\right] \mathrm{d} s \\
\leq & K A e^{p} \delta+\|X(t)\|_{0 k}\left\{|a(t)| \int_{t_{0}}^{t} e^{-\int_{s}^{t} a(v) \mathrm{d} v}\left[|b(s)|+\lambda_{1}(s)+\lambda_{2}(s)\right] \mathrm{d} s\right. \\
& \left.+|b(t)|+\lambda_{1}(t)+\lambda_{2}(t)\right\} \\
\leq & K A e^{p} \delta+\alpha\|X(t)\|_{0 k}, \quad t \in\left[t_{k},+\infty\right) .
\end{aligned}
$$

Hence, we have $\|X(t)\|_{0 k} \leq M \delta+\alpha\|X(t)\|_{0 k}$, that is, $\left.\left\|D_{H} X(t)\right\|\right\}$ for $t \in\left[t_{k},+\infty\right)$. Furthermore, it follows from $\|X(t)\|_{0 k} \leq(M / 1-\alpha) \delta$, where $\|X(t)\|_{0 k}=\max \{\|X(t)\|$,

$$
\begin{aligned}
\left\|X\left(t_{n}\right)\right\| \geq & \left\|\Phi\left(t_{k}\right)\right\| e^{-\int_{t_{k}}^{t_{n}} a(v) \mathrm{d} v}-e^{-\int_{t_{0}}^{t_{n}} a(v) \mathrm{d} v} \int_{t_{k}}^{t_{n}} e^{\int_{t_{0}}^{s} a(v) \mathrm{d} v}\left\|b(s) D_{H} X(s-\tau)+F(s, X(s), X(s-\tau))\right\| \mathrm{d} s \geq\left\|\Phi\left(t_{k}\right)\right\| e^{-\int_{t_{k}}^{t_{n}} a(v) \mathrm{d} v} \\
& -\|X(t)\|_{0 k} e^{-\int_{t_{0}}^{t_{n}} a(v) \mathrm{d} v} \int_{t_{k}}^{t_{n}} e^{\int_{t_{0}}^{s} a(v) \mathrm{d} v}\left[|b(s)|+\lambda_{1}(s)+\lambda_{2}(s)\right] \mathrm{d} s \\
> & \frac{1}{2} \delta e^{-p}-\frac{M}{1-\alpha} \delta e^{p} \frac{1-\alpha}{4 M e^{2 p}}=\frac{e^{-p}}{4} \delta
\end{aligned}
$$

which contradicts with (26). Hence, condition (16) is necessary for globally asymptotic stability of the trivial solution of (1). This completes the proof of Theorem 1 .

To illustrate our result, we give an example.

Example 1. In equation (1), let $a(t)=(1 / \sqrt{1+t})$, $b(t)=(1 / 8 \sqrt{1+t})$, and

$$
F(t, X(t), X(t-\tau))=\frac{1}{8 \sqrt{1+t}}[X(t)+X(t-\tau)] .
$$

By a straightforward computation, we can obtain

$$
|a(t)| \leq 1, \quad \int_{t_{0}}^{+\infty} a(s) \mathrm{d} s=+\infty, \quad \text { for } t \in I .
$$

In addition,

$$
\begin{aligned}
\left\|F\left(t, X_{1}, Y_{1}\right)-F\left(t, X_{2}, Y_{2}\right)\right\| & =\frac{1}{8 \sqrt{1+t}}\left\|X_{1}+Y_{1}-X_{2}-Y\right\|_{2} \\
& \leq \frac{1}{8 \sqrt{1+t}}\left(\left\|X_{1}-X_{2}\right\|+\left\|Y_{1}-Y_{2}\right\|\right) .
\end{aligned}
$$

Let $\lambda_{1}(t)=\lambda_{2}(t)=(1 / 8 \sqrt{1+t})$; then, condition $\left(\mathrm{A}_{2}\right)$ of Theorem 1 holds. By direct calculations, we have

$$
\begin{gathered}
\int_{t_{0}}^{t} e^{-\int_{s}^{t} a(v) \mathrm{d} v}\left[|b(s)|+\lambda_{1}(s)+\lambda_{2}(s)\right] \mathrm{d} s \\
\leq \frac{3}{8} e^{-2 \sqrt{1+t}} \int_{t_{0}}^{t} e^{2 \sqrt{1+s}} \frac{1}{\sqrt{1+s}} \mathrm{~d} s \\
\leq \frac{3}{8}\left[1-e^{2-2 \sqrt{1+t}}\right]<\frac{3}{8},
\end{gathered}
$$

$$
\begin{aligned}
& |a(t)| \int_{t_{0}}^{t} e^{-\int_{s}^{t} a(v) \mathrm{d} v}\left[|b(s)|+\lambda_{1}(s)+\lambda_{2}(s)\right] \mathrm{d} s \\
& \quad+|b(t)|+\lambda_{1}(t)+\lambda_{2}(t) \\
& \leq \frac{3}{8 \sqrt{1+t}}\left[1-e^{2-2 \sqrt{1+t}}\right]+\frac{3}{8 \sqrt{1+t}}<\frac{3}{4} .
\end{aligned}
$$

Let $\alpha=(3 / 4)$, and by (38)-(41), we find that conditions $\left(A_{1}\right)-\left(A_{3}\right)$ and (16) hold. Thus, (1) is globally asymptotically stable. 


\section{Conclusion}

Stability is one of the main problems encountered in applications and has recently attracted considerable attention. The fixed point method is an effective method to discuss the stability for the differential equation with unbounded delay or the differential equation with unbounded terms. In this paper, we investigate a class of neutral set-valued functional differential equations and obtain a criterion for the globally asymptotic stability theorem with necessary and sufficient conditions by the fixed point method. Finally, we verify the validity of the result by an example.

\section{Data Availability}

Data sharing is not applicable to this article as no data sets were generated or analyzed during the current study.

\section{Conflicts of Interest}

The authors declare that they have no conflicts of interest.

\section{Authors' Contributions}

All authors completed the paper together. All authors read and approved the final manuscript.

\section{Acknowledgments}

This paper was supported by the National Natural Science Foundation of China (11771115 and 11271106).

\section{References}

[1] T. A. Burton, "Liapunov functionals fixed points and stability by Krasnoselskii's theorem," Nonlinear Studies, vol. 9, pp. 181-190, 2001.

[2] T. A. Burton, "Stability by fixed point theory or Liapunov's theory: a Comparison," Fixed Point Theory, vol. 4, pp. 15-32, 2003.

[3] T. A. Burton, Stability by Fixed Point Theory for Functional Differential Equations, Dover Publications, New York, NY, USA, 2006.

[4] A. Abdelouaheb and D. Ahcene, "Fixed points and stability in linear neutral differential equations with variable delays," Nonlinear Analysis, vol. 74, pp. 2062-2070, 2011.

[5] T. A. Burton and T. Furumochi, "Asymptotic behavior of solutions of functional differential equations by fixed points theorem," Dynamic Systems and Applications, vol. 11, pp. 499-519, 2002.

[6] G. Chen, D. Li, O. van Gaans, and S. Verduyn Lunel, "Stability results for nonlinear functional differential equations using fixed point methods," Indagationes Mathematicae, vol. 29, no. 2, pp. 671-686, 2018.

[7] F. Ge and C. Kou, "Stability analysis by Krasnoselskii's fixed point theorem for nonlinear fractional differential equations," Applied Mathematics and Computation, vol. 257, pp. 308-316, 2015.

[8] C. H. Jin and J. W. Luo, "Fixed points and stability in neutral differential equations with variable delays," Proceedings of the American Mathematical Society, vol. 136, pp. 909-918, 2008.
[9] P. Manuel and S. Daniel, " $h$-Asymptotic stability by fixed point in neutral nonlinear differential equations with delay," Nonlinear Analysis, vol. 74, pp. 3926-3933, 2011.

[10] M. B. Mesmouli, A. Ardjouni, and A. Djoudi, "Study of the stability in nonlinear neutral differential equations with functional delay using Krasnoselskii-Burton's fixed-point," Applied Mathematics and Computation, vol. 243, pp. 492-502, 2014.

[11] R. Rao and S. Zhong, "Stability analysis of impulsive stochastic reaction-diffusion cellular neural network with distributed delay via fixed point theory," Complexity, vol. 2017, pp. 1-9, 2017.

[12] Y. N. Raffoul, "Stability in neutral nonlinear differential equations with functional delays using fixed-point theory," Mathematical and Computer Modelling, vol. 40, no. 7-8, pp. 691-700, 2004.

[13] C. S. Wang and Y. M. Li, "Krasnoselskii fixed point and exponential p-stability of neutral stochastic dynamic systems with time-varying delays," Chinese Journal of Applied Mechanics, vol. 36, no. 4, pp. 901-905, 2019.

[14] D. Zhao and D. Han, "Stability of linear neutral differential equations with delays and impulses established by the fixed points method," Nonlinear Analysis: Theory, Methods \& Applications, vol. 74, no. 18, pp. 7240-7251, 2011.

[15] V. Lakshmikantham, T. G. Bhaskar, and J. V. Devi, Theory of Set Differential Equations in Metric Spaces, Cambridge Scientific Publisher, Cambridge, UK, 2005.

[16] D. Azzam-Laouir and W. Boukrouk, "A delay second-order set-valued differential equation with Hukuhara derivatives," Numerical Functional Analysis and Optimization, vol. 36, no. 6, pp. 704-729, 2015.

[17] D. Azzam-Laouir and W. Boukrouk, "Second-order set-valued differential equations with boundary conditions," Journal of Fixed Point Theory and Applications, vol. 17, no. 1, pp. 99-121, 2015.

[18] A. Bashir and S. Sivasundaram, "Setvalued perturbed Hybrid integro-differential equations and stability in terms of two measures," Dynamic Systems and Applications, vol. 16, pp. 299-310, 2007.

[19] A. Bashir and S. Sivasundaram, "Basic results and stability criteria for set valued differential equations on time scales," Communications in Applied Analysis, vol. 11, pp. 419-428, 2007.

[20] Z. Drice, F. A. Mcrae, and J. V. Devi, "Set differential equations with causal operators," Mathematical Problems in Engineering, vol. 2, pp. 185-194, 2005.

[21] D. P. Nguyen, T. Q. Le, and T. T. Tran, "Stability criteria for set control differential equations," Nonlinear Analysis, vol. 69, pp. 3715-3721, 2008.

[22] T. G. Bhaskar and J. V. Devi, "Nonuniform stability and boundedness criteria for set differential equations," Applicable Analysis, vol. 84, no. 2, pp. 131-143, 2005.

[23] V. Lakshmikantham, S. Leela, and J. V. Devi, "Stability theory for set differential equations," Dynamics of Continuous, Discrete and Impulsive Systems Series A: Mathematical Analysis, vol. 11, pp. 181-190, 2004.

[24] N. T. Nguyen and T. T. Tran, "Stability of set differential euqations and applications," Nonlinear Analysis, vol. 71, pp. 1526-1533, 2009.

[25] U. Abbas, V. Lupulescu, D. O’Regan, and A. Younus, "Neutral set differential equations," Czechoslovak Mathematical Journal, vol. 65, no. 140, pp. 593-615, 2015.

[26] U. Abbas and V. Lupulescu, "Set functional differential equations," Communications on Applied Nonlinear Analysis, vol. 18, pp. 97-110, 2011. 
[27] A. Bashir and S. Sivasundaram, "Stability in terms of two measures for setvalued perturbed impulsive delay differential equations," Communications in Applied Analysis, vol. 12, no. 1 , pp. 57-68, 2008.

[28] J. V. Devi and A. S. Vatsala, "A study of set differential equations with delay," Dynamics of Continuous, Discrete and Impulsive Systems Series A: Mathematical Analysis, vol. 11, pp. 287-300, 2004.

[29] M. T. Malinowski, "Second type Hukuhara differentiable solutions to the delay set-valued differential equations," Applied Mathematics and Computation, vol. 218, no. 18, pp. 9427-9437, 2012.

[30] T. G. Bhaskar and J. V. Devi, "Stability criteria for set differential equations," Mathematical and Computer Modelling, vol. 41, no. 11-12, pp. 1371-1378, 2005.

[31] T. G. Bhaskar and J. V. Devi, "Set differential systems and vector Lyapunov functions," Applied Mathematics and Computation, vol. 165, pp. 539-548, 2005.

[32] G. Liu and J. Yan, "Global asymptotic stability of nonlinear neutral differential equation," Communications in Nonlinear Science and Numerical Simulation, vol. 19, no. 4, pp. 10351041, 2014. 